\title{
Aspectos epidemiológicos da tuberculose na população indígena Suruí, Amazônia, Brasil
}

\author{
Epidemiologic aspects of tuberculosis in the \\ Suruí Indians, Brazilian Amazon
}

\author{
Paulo Cesar Basta ${ }^{1}$, Carlos E.A. Coimbra Junior ${ }^{1}$, \\ Ana Lúcia Escobar ${ }^{2}$ e Ricardo Ventura Santos ${ }^{1}$
}

\begin{abstract}
RESUM0
A tuberculose permanece como prioridade de saúde pública no Brasil e atinge níveis preocupantes em certos segmentos sociais, como é o caso dos povos indígenas. 0 objetivo deste artigo é proceder a uma análise epidemiológica do banco de dados do Programa Municipal de Controle da Tuberculose em Cacoal, Rondônia, buscando-se caracterizar o perfil da doença no grupo indígena Suruí. Foi conduzida análise descritiva dos casos notificados entre 1975 e 2002. Os resultados evidenciam indicadores epidemiológicos alarmantes se comparados a de outros segmentos populacionais indígenas e não-indígenas. 0 coeficiente de incidência médio de tuberculose verificado nos Suruí no decênio 1991-2002 foi de 2518,9 por 100.000 habitantes. Foi observado que $45 \%$ dos casos foram em crianças < 15 anos e $63,3 \%$ eram do sexo masculino. Somente 43,2\% dos casos notificados tiveram confirmação baciloscópica. Não há registro de realização de testes com PPD, cultura ou exame histopatológico no período. Chama-se a atenção para a necessidade de implementação de medidas de prevenção e controle voltados especificamente para a realidade dos povos indígenas.
\end{abstract}

Palavras-chaves: Epidemiologia. Tuberculose. Índios sul-americanos. Serviços de saúde.

\begin{abstract}
Tuberculosis persists as a serious public health problem in Brazil. Prevalence rates are alarming in certain social groups, including indigenous peoples. This article presents an epidemiological analysis of records for the Suruí Indians available at the Tuberculosis Control Program in the Municipality of Cacoal, Rondônia. The study includes a descriptive statistical analysis of cases reported from 1975 to 2002. There is evidence that the Suruí have an increased risk of acquiring and dying from tuberculosis as compared to other indigenous groups in Rondônia as well as non-Indians. The average incidence coefficient for tuberculosis in the Suruí was 2518.9 per 100,000 inhabitants in the period 1991-2002. It was observed that $45 \%$ of the cases were diagnosed in children < 15 years old. Over half of the cases (63.3\%) were reported in men. Only $43.2 \%$ of the cases were confirmed by sputum microscopy. The use of PPD skin tests, histopathological exams or bacteriological culture were not reported throughout the period. Attention is called to the need for prevention and control measures specifically tailored to the reality of indigenous peoples.
\end{abstract}

Key-words: Epidemiology. Tuberculosis. South-American indians. Health Care System.

Atuberculose constitui prioridade de saúde pública no Brasil, caracterizando-se por apresentar elevada incidência e distribuição espacial heterogênea nas diferentes regiões do país, guardando estreita relação com as condições sócio-econômicas da população $0^{16}$.

Na Amazônia, onde vive cerca de $60 \%$ da população indígena do país, a incidência média da tuberculose atingiu 75,1 casos por 100.000 habitantes no período 1980-2000, a mais elevada dentre as regiões geopolíticas brasileiras ( a média nacional para esse período foi de 59,2) ${ }^{22}$. 0 impacto dessa endemia sobre as populações indígenas tem sido de grande magnitude, conforme apontam vários estudos realizados nas regiões amazônica e Centro-Oeste 34172526 . Análise recente evidenciou incidências de tuberculose de 286,8 e 326,8 casos por 100.000 habitantes

1. Escola Nacional de Saúde Pública da Fundação Oswaldo Cruz, Rio de Janeiro, RJ, Brasil. 2. Centro de Estudos em Saúde do Índio de Rondônia da Universidade Federal de Rondônia, Porto Velho, R0, Brasil.

Fontes de Financiamento: Fundação FORD e CNPq

Endereço para correspondência: Dr. Carlos E.A. Coimbra Jr. Rua Leopoldo Bulhões 1480. 21041-210 Rio de Janeiro, RJ.

Tel: 5521 2598-2683

e-mail: carlos_coimbrajr@gbl.com.br

Recebido em $\overline{16} / 12 / 2003$

Aceito em 2/6/2004 
para a população indígena na Amazônia, em 2000 e 2001, respectivamente ${ }^{14}$. Em Rondônia e regiões vizinhas, a tuberculose não apenas contribuiu para 0 significativo declínio populacional verificado em diversas etnias ao longo do século XX, como também permanece como proeminente causa de morbi-mortalidade ${ }^{11}$.

Esse estudo analisa aspectos da epidemiologia da tuberculose em um grupo indígena de Rondônia (os Suruí), que figura dentre aqueles nos quais têm sido verificadas as mais elevadas incidências da doença na região Norte ${ }^{14}$. A investigação baseou-se em informações disponibilizadas pelo Programa de Controle da Tuberculose (PCT) no município de Cacoal, Rondônia, e insere-se no bojo de um esforço de pesquisa que objetiva delinear o perfil epidemiológico das populações indígenas nessa região ${ }^{711} 12$.

\section{POPULAÇÃO E MÉTODOS}

Os Suruí constituem um dos grupos indígenas mais numerosos em Rondônia, totalizando cerca de 900 pessoas. Excetuando algumas famílias ou indivíduos que vivem em cidades próximas à terra indígena (em geral Cacoal ou Riozinho), a população está distribuída em dez aldeias situadas ao sul da Terra Indígena Sete de Setembro, localizada ao sudoeste do estado de Rondônia, próxima à divisa com Mato Grosso (aproximadamente $60-61^{\circ} \mathrm{W}$, 10-11 ${ }^{\circ} \mathrm{S}$ ). Em relação a sua inserção no subsistema de atenção à saúde indígena, os Suruí estão jurisdicionados ao Pólo-Base Cacoal, Distrito Sanitário Especial Indígena Vilhena, com sede na cidade de Cacoal. A etnia Suruí corresponde a cerca de 90\% da demanda atendida por esse Pólo.

Historicamente, a tuberculose exerceu grande impacto sobre a população Suruí, ocasionando grave epidemia que se seguiu pouco após 0 estabelecimento do contato permanente do grupo com a frente de atração coordenada pela Fundação Nacional do Índio (FUNAI), no início dos anos $1970^{56}$.

Os registros de casos de tuberculose para o período 19782002 foram obtidos junto ao PCT no município de Cacoal. Complementarmente, também foram consultados os registros remanescentes do extinto serviço de saúde da Fundação Nacional do Índio ( FUNAI), em Cacoal, para o período 1975-1988. Com base nesse banco de dados, foi construída uma série histórica de casos de tuberculose entre os Suruí para o período 19752002. No entanto, devido a lacunas de informações para os anos de 1977 e 1982-1984, optou-se por aprofundar a análise para 0 período 1991-2002. Para a determinação dos coeficientes de incidência, foram utilizados os dados demográficos disponíveis no Sistema de Informações de Saúde Indígena (SIASI) e, para as décadas de 1970-1980, censos anteriormente realizados pelos autores ${ }^{6}$. Năo foi possível avaliar os critérios através dos quais foram realizados os diagnósticos de tuberculose e a determinação das formas clínicas, especialmente em crianças.

\section{RESULTAD0S}

Adespeito das lacunas de dados verificadas para alguns anos, notam-se elevadíssimas taxas de incidência por tuberculose entre os Suruí ao longo das três últimas décadas (Tabelas 1 e 2).
Tabela 1 - Casos de tuberculose, coeficientes de incidência e proporção de casos confirmados por baciloscopia de escarro entre os Suruí, Rondônia (1975-1990).

\begin{tabular}{|c|c|c|c|c|c|c|}
\hline Ano & População & Total de & Inc/ & Casos $\left(n^{0}\right)$ & $\% \mathrm{BK}+$ & Inc/ \\
\hline & & casos & 100.000 hab & $\mathrm{BK}+$ & & 100.000 hab BKt \\
\hline 1975 & 184 & 16 & 8695,7 & - & - & \\
\hline 1976 & 199 & 1 & 502,5 & - & - & - \\
\hline 1978 & 232 & 2 & 862,1 & 1 & 50,0 & 413,0 \\
\hline 1979 & 251 & 2 & 796,8 & 1 & 50,0 & 456,1 \\
\hline 1980 & 266 & 2 & 751,9 & - & - & - \\
\hline 1981 & 282 & 9 & 3191,5 & 3 & 32,3 & 1063,8 \\
\hline 1985 & 354 & 1 & 282,5 & - & - & - \\
\hline 1986 & 373 & 2 & 536,2 & - & - & - \\
\hline 1987 & 394 & 1 & 253,8 & - & - & - \\
\hline 1988 & 415 & 1 & 241,0 & - & - & - \\
\hline 1989 & 437 & 2 & 457,7 & 2 & 100,0 & 457,7 \\
\hline 1990 & 461 & 2 & 433,8 & 2 & 100,0 & 433,8 \\
\hline Total & 3664 & 41 & $1118,9^{*}$ & 9 & 21,9 & $245,6^{*}$ \\
\hline
\end{tabular}

Tabela 2 - Casos de tuberculose, coeficientes de incidência e proporção de casos confirmados por baciloscopia de escarro entre os Suruí, Rondônia (1991-2002).

\begin{tabular}{lcccrrc}
\hline Ano & População & Total de & $\begin{array}{c}\text { Inc/ } \\
\text { casos }\end{array}$ & $\begin{array}{c}\text { Casos }\left(\mathrm{n}^{0}\right) \\
\text { 10000 hab }\end{array}$ & $\begin{array}{c}\text { BK+ } \\
\text { BKt }\end{array}$ & $\begin{array}{c}\text { Incl } \\
100.000 \text { hab BK+ }\end{array}$ \\
\hline 1991 & 486 & 11 & 2263,4 & 6 & 54,5 & 1234,6 \\
1992 & 512 & 28 & 5468,8 & 7 & 25,9 & 1367,2 \\
1993 & 539 & 30 & 5565,9 & 10 & 33,3 & 1855,3 \\
1994 & 568 & 13 & 2288,7 & 3 & 23,1 & 528,2 \\
1995 & 599 & 16 & 2671,1 & 10 & 62,5 & 1669,5 \\
1996 & 631 & 17 & 2694,1 & 5 & 29,4 & 792,4 \\
1997 & 665 & 12 & 1804,5 & 6 & 50,0 & 902,3 \\
1998 & 700 & 10 & 1428,6 & 6 & 60,0 & 857,1 \\
1999 & 738 & 11 & 1490,5 & 4 & 36,4 & 542,0 \\
2000 & 778 & 14 & 1799,5 & 9 & 64,3 & 1156,8 \\
2001 & 820 & 17 & 2073,2 & 8 & 47,1 & 975,6 \\
2002 & 864 & 20 & 2314,8 & 12 & 63,2 & 1388,9 \\
\hline Total & 7900 & 199 & $2518,9 *$ & 86 & 43,2 & $1088,6^{*}$ \\
\hline *Coeficiente médio no período. &
\end{tabular}

Para o período de 1975-1990, 0 coeficiente médio de incidência foi de 1118,9 por 100.000 habitantes e de 245,6 por 100.000 para os casos confirmados através da baciloscopia. Já para 0 decênio 1991-2002, o coeficiente de incidência médio verificado foi de 2518,9 por 100.000 habitantes e de 1088,6 por 100.000 para os confirmados através da baciloscopia.

Considerando-se os casos notificadosa partir de 1991, verificouse incidência de tuberculose acima de 5.000 por 100.000 habitantes nos anos de 1992 e 1993. No período 1994-1998, verificou-se lenta redução, atingindo taxas acima de 1.000 por 100.000 habitantes, quando voltou a crescer, ultrapassando 2.000 por 100.000 habitantes no ano de 2002 (Figura 1). Considerando-se a proporção de casos de tuberculose pulmonar diagnosticados com base em exame baciloscópico do escarro, o ano de 1994 apresentou o pior desempenho, com apenas 23,1\% (Tabela 2). A proporção de casos confirmados através da baciloscopia foi igual ou superior a 50\% em seis anos (1991, 1995, 1997, 1998, 2000 e 2002).

Cerca de 57\% dos casos notificados entre 1991 e 2002 não tiveram comprovação baciloscópica (Tabela 3). Há 


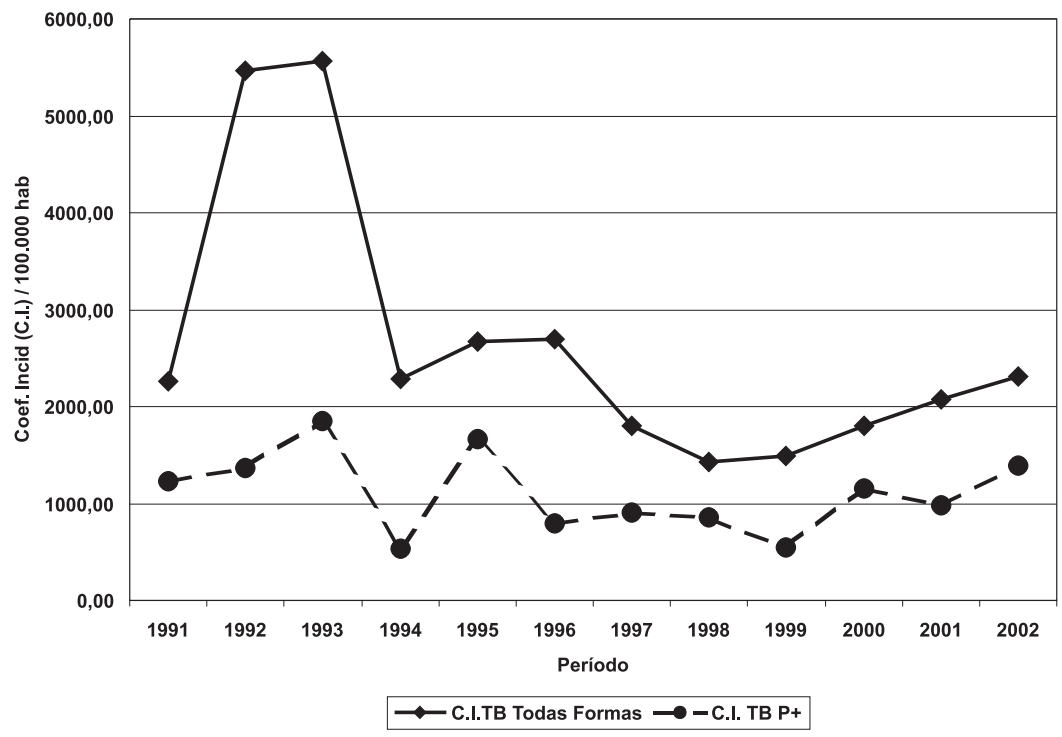

Figura 1 - Incidência de tuberculose (por 100.000 hab), todas as formas e formas pulmonares com baciloscopia de escarro positiva, população Suruí, 1991-2002.

Tabela 3 - Casos de tuberculose entre os Suruí, Rondônia, segundo faixa etária, sexo eproporção de confirmados por baciloscopia (BK+), 1991-2002.

\begin{tabular}{lcrrrr}
\hline Faixa etária & & \multicolumn{2}{c}{ Sexo } & & \\
\cline { 3 - 4 } (anos) & & masc & fem & Total & Casos BK+ \\
\hline $00 \mid-01$ & casos & 3 & 3 & 6 & - \\
& $(\%)$ & 50,0 & 50,0 & 100,0 & - \\
$01 \mid-05$ & casos & 23 & 19 & 42 & 2 \\
& $(\%)$ & 54,8 & 45,2 & 100,0 & 4,8 \\
$05 \mid-10$ & casos & 14 & 5 & 19 & 4 \\
& $(\%)$ & 73,7 & 26,3 & 100,0 & 21,1 \\
$10 \mid-15$ & casos & 15 & 7 & 22 & 15 \\
& $(\%)$ & 68,2 & 31,8 & 100,0 & 68,2 \\
$15 \mid-20$ & casos & 30 & 3 & 33 & 28 \\
& $(\%)$ & 90,9 & 9,1 & 100,0 & 84,8 \\
$20 \mid-50$ & casos & 27 & 27 & 54 & 29 \\
& $(\%)$ & 50,0 & 50,0 & 100,0 & 53,7 \\
$\geq 50$ & casos & 14 & 9 & 23 & 8 \\
& $(\%)$ & 60,9 & 39,1 & 100,0 & 34,8 \\
\hline Total & casos & 126 & 73 & 199 & 86 \\
& $(\%)$ & 63,3 & 36,7 & 100,0 & 43,2 \\
\hline
\end{tabular}

registro de 9\% dos casos diagnosticados em crianças < 10 anos de idade através da baciloscopia. A radiografia foi utilizada em 91\% dos casos na investigação diagnóstica no mesmo grupo de idade. 0 melhor índice de casos confirmados microscopicamente foi verificado na faixa de 15-20 anos de idade (85\%). Năo houve, para todo 0 período analisado, registro de envio de lâminas com esfregaços de escarro para controle de qualidade junto a qualquer unidade de referência estadual ou federal. Tampouco foi verificado registro de utilização do PPD, cultura ou exame histopatológico no diagnóstico dos casos notificados.

Entre 1991 e 2002 foram notificados 221 casos de tuberculose em indígenas em Cacoal. Desse total, 199 (90\%) eram Suruí (incluindo uma mulher e uma criança Cinta Larga que vivem entre estes) , 20 ( 9\%) eram Cinta Larga e 2 (0,9\%) indivíduos pertenciam a outras etnias.
À exceção de um único caso de tuberculose pleural, em um indivíduo do sexo masculino com 22 anos de idade, os demais se apresentaram sob a forma pulmonar.

De um total de 199 casos notificados para o período 19912002, 126 (63,3\%) foram em homens e 73 (36,7\%) em mulheres $\left(\chi^{2}=14,1 ; 1\right.$ g.l.; $\left.p<0,01\right)$. As diferenças entre os sexos foram particularmente pronunciadas nas faixas etárias de 5-10 anos (73,7\% dos casos em homens) e 15-20 anos ( 90,9\% dos casos em homens). Nota-se também que aproximadamente metade dos casos de tuberculose (45\%) foi notificada em crianças < 15 anos de idade.

A distribuição de casos de tuberculose não se deu de forma homogênea entre as dez aldeias Suruí. Houve concentração na aldeia da Linha 14 (38,2\% das notificações no período), seguida pelo conjunto dos quatro aldeamentos que compõem a Linha $11(19,1 \%)$. As demais aldeias apresentaram as seguintes proporções de casos: Linha 9 (14,1\%), Linha 12 (12,1\%), Linha 8 (5,5\%) e Linha 10 (3,5\%). 0 grupo que tem residência na localidade de Riozinho apresentou 7,5\% do total de casos.

Dos 199 casos com desfecho conhecido, 13 (6,5\%) abandonaram 0 tratamento e 181 (91\%) tiveram alta por cura. Foram registrados três (1,5\%) óbitos e um ( $0,5 \%)$ único caso de falência de tratamento. Năo há registro de mudança de diagnóstico no PCT.

\section{DISCUSSÃ0}

A tuberculose instalou-se entre os Suruí no início da década de 1970 e, desde então, vem se mantendo altamente endêmica, apresentando coeficientes de incidência muito superiores àqueles verificados na população regional. Para 0 Estado de Rondônia, em 1999 foi verificado coeficiente de incidência de 43 casos por 100.000 habitantes ${ }^{23}$. Dentre os indígenas do Estado, no período de 1992-1998, os coeficientes médios de incidência eram da ordem de 1.000 casos por 100.000 
habitantes ${ }^{11}$, enquanto para os Pakaanóva (Wari') os coeficientes foram de 893,6 e 654,9 por 100.000 habitantes em 1995 e 1996, respectivamente ${ }^{10}$.

No presente estudo verificou-se maior concentração de casos em homens do que em mulheres, da ordem de 1,7:1. Por um lado, se essa distribuição é compatível com o observado na população brasileira em geral, onde os homens respondem por mais da metade dos casos de tuberculose, por outro diverge daquela verificada entre outras etnias de Rondônia, nas quais não foram detectadas diferenças entre os sexos ${ }^{11}{ }^{12}$. A relativa proximidade das aldeias Suruí com Cacoal e Riozinho facilita sobremaneira a intensa circulação, principalmente de homens, nesses centros urbanos. Esse fator pode estar relacionado ao delineamento de um perfil epidemiológico da tuberculose entre os Suruí divergente daquele observado em outros grupos indígenas da região no tocante à proporção de casos entre homens e mulheres.

A distribuição espacial dos casos de tuberculose entre os Suruí não é homogênea entre as aldeias. Há nítida concentração nas aldeias conhecidas por Linha $11(19,1 \%)$ e Linha 14 (38,2\%). É possível que, em parte, essa concentração possa estar associada ao fato de se tratarem das duas aldeias mais populosas, nas quais se encontra cerca de $60 \%$ da população.

Entre os Suruí, a tuberculose atinge largamente crianças $<15$ anos ( $45 \%$ dos casos), o que diverge do observado para a população em geral, que oscila em torno de 10-15\% para essa faixa etária ${ }^{1619}$. É expressivo o número de casos notificados em crianças $<5$ anos (aproximadamente $1 / 4 \mathrm{do}$ total). Chama atenção a existência de dois casos de tuberculose em crianças $<5$ anos de idade com confirmação atavés de baciloscopia de escarro. 0 diagnóstico da tuberculose na infância é especialmente complicado, principalmente pela dificuldade de se obter escarro. Não obstante, há critérios relativamente bem estabelecidos para o diagnóstico de tuberculose nesse grupo etário, que se baseiam em sistema de pontuação que leva em consideração, além de dados clínicos, a história de contato com doente bacilífero, PPD e radiografia de tórax, dentre outros ${ }^{2728}$. 0 Ministério da Saúde ${ }^{20}$ recomenda a aplicação de um modelo de pontuação para 0 diagnóstico de tuberculose na infância, que apresenta elevada sensibilidade e especificidade ${ }^{24}$.

0 banco de dados analisado contém inúmeras lacunas que impedem 0 desenvolvimento de análises estatísticas mais robustas, visando dentre outros objetivos a caracterização de tendências. Durante largo período de tempo não havia registros de idade confiáveis para os indígenas. Por exemplo, durante análise da série histórica, foi detectada a presença de uma paciente Suruí com vários registros repetidos ao longo de seis anos, mas com a idade notificada sempre a mesma.

Chama atenção 0 fato de não terem sido realizados testes com PPD, cultura ou exame histopatológico no período estudado. Também em número expressivo (24,6\%) de casos não há registro de utilização de radiografia na investigação diagnóstica. A elevada proporção de diagnósticos realizados com base em critérios unicamente clínicos já foi apontada em trabalho anterior que incluiu a totalidade dos casos de tuberculose em indígenas de
Rondônia no período 1992-1998, tendo sido observado que em apenas 38\% destes houve confirmação baciloscópica ${ }^{11}$. 0 estabelecimento de diagnóstico eminentemente clínico da tuberculose foge das diretrizes consagradas, tanto no Brasil como no exterior, para 0 controle dessa endemia ${ }^{1830}$, acarretando risco ao paciente e ônus ao serviço ( tratamento prolongado, consultas de controle, exames complementares e erros).

A larga utilização de parâmetros unicamente clínicos no diagnóstico da tuberculose verificada entre os Suruí, por parte do PCT-Cacoal, apresenta complicações adicionais quando se leva em consideração a ocorrência de outras doenças infecciosas pulmonares endêmicas na região, como a paracoccidioidomicose ${ }^{9}$. Já foi apontado que a elevada letalidade devido a essa micose verificada entre os Suruí pode estar associada ao estabelecimento de diagnósticos tardios. Não raro, os doentes são inicialmente submetidos a tratamento para tuberculose, sem confirmação baciloscópica ${ }^{13} 29$. Apesar dos muitos casos de paracoccidioidomicose conhecidos entre os Suruí e reportados na literatura, muitos dos quais submetidos a tratamento para tuberculose, vale destacar que não houve registro de mudança de diagnóstico na série analisada.

Para os Suruí, os dados do PCT indicam um alto (91\%) índice de cura, superando aqueles $(84 \%)^{11}$ verificados para a população indígena de Rondônia e para a população geral brasileira ( cerca de $80 \%)^{1516}$. Nos dias atuais, possíveis fatores favorecedores desse desempenho incluem a distribuição da medicação aos pacientes sem interrupção e 0 comparecimento dos Suruí às consultas, graças à equipe do pólo-base ao qual esses indígenas estão jurisdicionados. Por outro lado, os tratamentos são autoadministrados, sem supervisão direta. Deve-se ressaltar, no entanto, que os dados sobre desempenho do programa não garantem que, efetivamente, esteja acontecendo plena adesão ao esquema terapêutico preconizado por parte do paciente. De volta à aldeia, após a consulta, não há como assegurar que os pacientes realmente tomem a medicação na forma prescrita, limitando-se 0 PCT a registrar como adesão aquele que comparece a consulta e que declara estar tomando a medicação.

A alta (45\%) proporção de casos em crianças menores de 15 anos, com diagnóstico baseado em larga escala apenas em exame clínico e radiografia de tórax ( sem investigações adicionais), associada ao baixo índice de baciloscopias positivas e a falta de supervisão no tratamento dispensado, podem levantar questionamentos importantes em relação ao desempenho do PCT, especialmente no que diz respeito aos indicadores de cura, mudança de diagnóstico e abandono. Para um melhor entendimento desta situação seria necessário desenvolver um estudo para 0 acompanhamento dos casos que iniciaram tratamento, com 0 objetivo de identificar questões de ordem microbiológica ( associadas ao bacilo), sóciocultural ( associadas aos pacientes e profissionais de saúde) e organizacional (associadas ao serviço de saúde) relacionadas a eficácia das ações conduzidas pelo programa.

Apesar das lacunas identificadas na base de dados, foi possível derivar algumas conclusões importantes relacionadas à epidemiologia da tuberculose entre os Suruí e à atuação dos serviços de saúde. Aaltíssima incidência de tuberculose verificada 
em crianças e adolescentes revela a ocorrência de infeccão recente por contato com tuberculosos baciliferos, o que sugere que o controle de contatos não está sendo realizado ${ }^{1721}$. Os diagnósticos tendem a ser feitos unicamente em função da demanda, não havendo exame rotineiro de sintomáticos respiratórios ou busca ativa de comunicantes nas aldeias. Tampouco tem sido feita quimioprofilaxia dos infectados sob risco de adoecer, o que contraria recomendações amplamente divulgadas por organizaçôes nacionais e internacionais ${ }^{18} 1930$.

Experiências desenvolvidas entre povos indígenas de outras regiões do Brasil, enfocando a estratégia de tratamento ambulatorial associada ao tratamento domiciliar assistido, têm produzido resultados promissores, além de estreitar as bases para cooperação entre autoridades sanitárias municipais, agentes indígenas de saúde e outras organizações vinculadas à prestação de servicos de saúde aos povos indígenas ${ }^{17}$. Promover melhorias no PCT é uma etapa fundamental para reverter 0 preocupante quadro epidemiológico da tuberculose entre os Suruí.

\section{AGRADECIMENTOS}

Aos enfermeiros Dalvanira G. Costa e Ivani C. Gromann da PACA (Proteção Ambiental Cacoalense), João Batista Ramos Pordeus da Casa de Saúde do Índio de Riozinho eMaria da Conceiç̃o Alves Cunha da Secretaria Municipal de Saúde de Cacoal, pelo apoio e acesso aos dados. Ao enfermeiro Jesem Douglas Y. Orellana pela ajuda na construção do banco de dados. Ao Professor Reinaldo Souza-Santos da Escola Nacional de Saúde Pública pela valiosa contribuição nas análises estatásticas e discussão do texto. Essa pesquisa foi financiada com recursos da Fundacão Ford, através do Centro de Estudos em Saúde do Índio de Rondônia, da Universidade Federal de Rondônia.

\section{REFERÊNCIAS BIBLIOGRÁFICAS}

1. Afiune JB, Ide-Neto J. Diagnóstico da tuberculose pulmonar escarro negativo. Jornal de Pneumologia 19: 37-41, 1993.

2. Amarante JM, Costa VLA. A tuberculose nas comunidades indígenas brasileiras na virada do século. Boletim de Pneumologia Sanitária 8: 5-12, 2000.

3. Baruzzi RG, Barros VL, Rodrigues D, Souza ALM, Pagliaro H. Saúde e doença em índios Panará (Kreen-Akarôe) após vinte e cinco anos de contato com o nosso mundo, com ênfase na ocorrência de tuberculose (Brasil Central). Cadernos de Saúde Pública 17: 407-412, 2001.

4. Buchillet D, Gazin P. A situação da tuberculose na população indígena do alto rio negro (Estado do Amazonas, Brasil). Cadernos de Saúde Pública 14: 181-185, 1998.

5. Chiappino, J. The Brazilian indigenous problem and policy: the Aripuanã park, document no 19 Copenhagen: International Work Group for Indigenous Affairs, 1975.

6. Coimbra Jr. CEA. From shifting cultivation to coffee farming: the impact of change on the health and ecology of the Suruí Indians in the Brazilian Amazon. Ph.D. dissertation. Indiana University, Bloomington, 1989.

7. Coimbra Jr CEA, Santos RV, Escobar AL. Saúde indígena em Rondônia na década de 90. In: Ricardo CA ( org) Povos Indígenas no Brasil 1996-2000, Instituto Socioambiental, São Paulo, p. 591-593, 2000.

8. Coimbra Jr CEA, Wanke B, Santos RV, Valle ACF, Costa RLB, Zancopé-Oliveira RM. Paracoccidioidin and histoplasmin sensitivity in Tupí-Mondé Amerindian populations from Brazilian Amazonia. Annals of Tropical Medicine and Parasitology 88: 197-207, 1994.

9. Coutinho ZF, Silva D, Lazera M, Petri V, Oliveira RM, Sabroza PC, Wanke, B. Paracoccidioidomycosis mortality in Brazil (1980-1995). Cadernos de Saúde Pública 18: 1441-1454, 2002.
10. Escobar AL. Epidemiologia da tuberculose na população indígena Pakaanóva (Wari') , Estado de Rondônia, Brasil. Tese de Doutorado, Escola Nacional de Saúde Pública, Fundação Oswaldo Cruz, Rio de Janeiro, 2001.

11. Escobar AL, Coimbra Jr CEA, Camacho LA, Portela MC. Tuberculose em populações indígenas de Rondônia, Amazônia, Brasil. Cadernos de Saúde Pública 17: 285-298, 2001

12. Escobar AL, Coimbra Jr CEA, Camacho LA, Santos RV. Tuberculin reactivity and tuberculosis epidemiology in the Paakanova (Wari') Indians of Rondônia, Southwestern Brazilian Amazon. International Journal of Tuberculosis and Lung Diseases 8: 45-51, 2004.

13. Forjaz MHH, Fischman 0, Camargo ZP, Vieira Filho JPB, Colombo AL. Paracoccidioidomicose em índios brasileiros da tribo Suruí: estudo clínicolaboratorial de 2 casos. Revista da Sociedade Brasileira de Medicina Tropical 32: 571-575, 1999.

14. Garnelo L, Macedo G, Brandão LC. Os povos indígenas e a construção das políticas de saúde no Brasil. Organização Pan-Americana de Saúde, Brasília - DF, 2003.

15. Gerhardt G, Ribeiro SN. Eficiência do tratamento da tuberculose no Brasil. Informe Epidemiológico do Sistema Único de Saúde 4: 95-98, 1995.

16. Hijjar MA. Controle das doenças endêmicas no Brasil - Tuberculose. Revista da Sociedade Brasileira de Medicina Tropical 27 ( supl 3) :23-36, 1994.

17. Marques AMC, Cunha RV. A medicação assistida e os índices de cura de tuberculose e de abandono de tratamento na população indígena GuaraniKaiwá no município de Dourados, Mato Grosso do Sul, Brasil. Cadernos de Saúde Pública 19:109-118, 2003.

18. Ministério da Saúde. Fundação Nacional de Saúde, Centro Nacional de Epidemiologia, Coordenação Nacional de Pneumologia Sanitária, Sociedade Brasileira de Pneumologia e Tisiologia. I Consenso Brasileiro de Tuberculose. Jornal de Pneumologia 23: 281-342, 1997.

19. Ministério da Saúde. Plano nacional de controle da tuberculose. Secretaria de Políticas de Saúde, Departamento de Gestão de Políticas Estratégicas de Saúde, Coordenação Nacional de Pneumologia Sanitária. Fundação Nacional de Saúde, Centro Nacional de Epidemiologia, Centro de Referência Hélio Fraga, Brasília, 1999.

20. Ministério da Saúde. Manual de controle de tuberculose. Centro Nacional de Epidemiologia/Coordenação do Programa Nacional de Imunizações, Brasília, 2001.

21. Natal S. Tuberculose na criança. Boletim de Pneumologia Sanitária 8: $21-25,2000$

22. Natal S, Oliveira MPJ, Hijjar MA. Mortalidade e letalidade por tuberculose no Brasil - 1980 a 2000. Jornal de Pneumologia 28 (supl. 2) : s28, 2002.

23. Ruffino-Neto A. Tuberculose: a calamidade negligenciada. Revista da Sociedade Brasileira de Medicina Tropical 35: 51-58, 2002.

24. Sant'anna CC, Orfaliais CTS, March MFBP. A retrospective evaluation of a score system adopted by the Ministry of Health, Brazil in the diagnosis of pulmonary tuberculosis in childhood: A case control study. Revista do Instituto de Medicina Tropical de São Paulo 45: 103-105, 2003.

25. Santos RV, Coimbra Jr CEA. Cenários e tendências da saúde e da epidemiologia dos povos indígenas no Brasil. In: Coimbra Jr CEA, Santos RV, Escobar AL( orgs) Epidemiologia e saúde dos povos indígenas no Brasil, Editora FIOCRUZ, Rio de Janeiro, p.13-48, 2003.

26. Sousa AO, Salem JI, Lee FK, Verçosa MC, Cruaud P, Bloom BR, Lagrange PH, David HL. An epidemic of tuberculosis with a high rate of tuberculin anergy among a population previously unexposed to tuberculosis, the Yanomami Indians of the Brazilian Amazon. Proceeding of the National Academy of Sciences of the United States of America 94: 13227-13232, 1997.

27. Stegen G, Jones K, Kaplan P. Criteria for guidance in the diagnosis of tuberculosis. Pediatrics 43: 260-263, 1969.

28. Tidjani 0 , Amedome A, ten Dam HG. The protective effect of BCG vaccination of the newborn against childhood tuberculosis in an African community. Tubercle 67: 269-281, 1986.

29. Valle ACF, Coimbra Jr CEA, Llinares FTB, Montoiro PCF, Guimarães MRC. Paracoccidioidomicose entre o grupo indígena Suruí de Rondônia, Amazônia (Brasil). Registro de caso. Revista do Instituto de Medicina Tropical de São Paulo 33: 407-411, 1991.

30. World Health Organization. Treatment of tuberculosis: Guidelines for national programmes. Geneva, 2003. 